\title{
Cerebrovascular contribution to dementia development after traumatic brain injury: promises and problems
}

\author{
Lyndsey Collins-Praino ${ }^{1}$, Frances Corrigan ${ }^{2}$ \\ ${ }^{1}$ Discipline of Anatomy and Pathology, Adelaide Medical School, University of Adelaide, Adelaide, South Australia; ${ }^{2}$ School of Health Sciences, \\ University of South Australia, Adelaide, South Australia \\ Correspondence to: Frances Corrigan, PhD. Head Injury Laboratory, School of Health Sciences, University of South Australia, Adelaide 5000, \\ Australia. Email: frances.corrigan@unisa.edu.au. \\ Comment on: Ramos-Cejudo J, Wisniewski T, Marmar C, et al. Traumatic Brain Injury and Alzheimer's Disease: The Cerebrovascular Link. \\ EBioMedicine 2018;28:21-30.
}

Submitted Sep 28, 2018. Accepted for publication Oct 10, 2018.

doi: $10.21037 / \mathrm{atm} .2018 .10 .22$

View this article at: http://dx.doi.org/10.21037/atm.2018.10.22

Traumatic brain injury (TBI) is a leading cause worldwide of death and disability. Increasing evidence suggests that a TBI is not just an acute event, but sets in motion a number of ongoing pathological cascades that increase the risk of later developing dementia. In support of this, the most recent retrospective cohort study to investigate this link by Barnes et al., utilised the Veterans Health Administration health care database. It was found that the adjusted hazards ratio for a dementia diagnosis were 3.77 for those with a moderate to severe TBI and 2.36-2.51 for those who had a mild TBI (mTBI), depending on whether loss of consciousness was observed at the time of impact (1). This highlights that there is two-four fold increased risk of developing dementia following TBI and that risk increases with the severity of the initial impact [see for review (2)]. These epidemiological studies are supported by postmortem studies showing that TBI increases the deposition of pathological proteins associated with neurodegenerative disease. Long-term survivors of a single moderate-severe TBI were found to have more widespread A $\beta$ plaques and a higher incidence of neurofibrillary tangles (NFTs) compared to age-matched controls (3).

The type of dementia associated with TBI remains less clear and may depend on the type of initial insult involved. Traditionally moderate-severe TBI has been associated with an increased risk of Alzheimer's disease (AD) and repeated concussive/sub-concussive impacts with chronic traumatic encephalopathy (CTE) (4), but there is also evidence to suggest that TBI associated dementia may be its own distinct entity with features that may overlap with other neurodegenerative diseases (3). In support of this, the pattern of localisation of NFTs seen following trauma is distinct to that of other neurodegenerative diseases. Following TBI, NFTs are typically seen at the depths of the sulci and are commonly seen perivascularly, first appearing in the superficial cortical layers (4). In contrast, in $\mathrm{AD}$, NFTs first appear in the transentorhinal regions before spreading to the cortex, with involvement of the deeper cortical layers (5). This localisation of tau post-trauma is seen both following a history of repeated milder impacts, where it forms the key basis for the post-mortem diagnosis of CTE (4), as well as following single moderate-severe TBI (3). In regards to $A \beta$ pathology, differences have been also reported between survivors of a moderate-severe and more mild injuries. In long-term (1-47 years post-injury) survivors of a single moderate severe TBI, widespread, predominantly fibrillary $\mathrm{A} \beta$ plaques are noted within the cortex in $28 \%$ of individuals, with spread to the entorhinal cortex in a few cases (3). This is in line with the spread of $\mathrm{A} \beta$ in $\mathrm{AD}$, which begins in the isocortex before spreading to regions of the limbic system, other subcortical regions and finally the brainstem. However, notable hippocampal sparing was seen in the TBI survivors compared to traditional AD (3). Contrastingly, in CTE cases, which may be more related to repeated concussive/sub-concussive impacts, only $50 \%$ have $\mathrm{A} \beta$ pathology, with no consistent description of regional distribution to date, with $A \beta$ not required for disease development, although it is thought to 
play a role in accelerating disease progression (4).

Thus, considering whether the pattern of neuropathological change and, consequently, neurodegenerative disease is similar or distinct depending on the nature of the original injury is an important element not considered within the Ramos-Cejudo et al. paper. Indeed, TBI has been characterised as a highly complex disease, with heterogeneity driven by the differences in terms of severity and location of the initiating impact, whether the injury is predominantly focal versus diffuse and whether multiple or single injuries have occurred (6). The potential impact of this heterogeneity on subsequent molecular alterations is not considered by Ramos-Cejudo and colleagues, who instead discuss the potential link between TBI and $\mathrm{AD}$ as if all injuries are created equal. This may mask important distinctions that may have significant implications for biomarker identification. For example, if the type of dementia that follows TBI is dependent upon the initial nature of the injury, then it logically follows that the biomarkers of interest for predicting relative risk of each of these dementias may also be distinct. Further research is needed to determine whether the pathological processes that drive neurodegeneration post-TBI share key overlapping pathological processes, regardless of the nature of the initiating insult, or whether they are distinct, as these investigations will inform development of appropriate biomarker panels.

Nonetheless, it is clear that a history of TBI can be linked to accumulation of pathological proteins, and ongoing neuronal loss. The mechanisms linking TBI to later dementia has yet to be fully elucidated. Hypotheses include a reduction in neuronal reserve precipitating earlier age of onset of dementia, chronic persistent neuroinflammation and ongoing white matter injury (3). Ramos-Cejudo et al. argue that the key mechanistic link between TBI and dementia is ongoing dysfunction of the cerebrovascular system associated with accumulation of pathological A $\beta$ and tau. Importantly, there is key evidence supporting this supposition, which suggests that disruption to cerebral blood flow and alterations of the blood-brain barrier (BBB) are early events in the onset of dementia and this is well described within the original article. A $\beta$ pathology has been shown to lead to multiple forms of secondary vascular damage, including microinfarcts, microbleeds and white matter lesions (7). While the mechanisms behind this remain to be elucidated, oligomeric species of $\mathrm{A} \beta$ have been shown to lead to a cascade of neurovascular stress events, including oxidative stress and activation of caspase-mediated apoptotic pathways in endothelial cells (8). Similarly, cerebrovascular tau oligomers have been found to be elevated in two forms of dementia, $\mathrm{AD}$ and progressive supranuclear palsy (PSP), where they preferentially associate with vascular endothelial cells Tau pathology-dependent remodelling of cerebral arteries precedes Alzheimer's disease-related microvascular cerebral amyloid angiopathy (9). Tau pathology has recently been shown to lead to early remodelling of leptomeningeal arteries, preceding microvascular cerebral amyloid angiopathy, in the gyrus frontalis medialis and the hippocampus in $\mathrm{AD}$ (10). Interestingly, BBB dysfunction has been shown to emerge at the same time as perivascular tau deposition around major hippocampal blood vessels, an effect that can be blocked by suppressing tau expression using doxycycline (11). This, coupled with the finding that $\mathrm{BBB}$ dysfunction occurs even in tauopathies without $\mathrm{A} \beta$ pathology, leads to the compelling hypothesis that tau pathology alone may drive cerebrovascular dysfunction. In support of this, high CSF levels of neuroinflammation and cerebrovascular dysfunction were recently reported to be associated with increased CSF levels of both total and phosphorylated tau in $\mathrm{AD}$, while associations between these markers and CSF levels of A $\beta$ were less clear (12). Taken together, this body of evidence presents a compelling case that $\mathrm{A} \beta$ and tau pathology, both of which are known to be elevated after TBI, can drive cerebrovascular dysfunction and, consequentially, the emergence of dementia.

However, given that the central tenant of 'Traumatic Brain Injury and Alzheimer's Disease: The Cerebrovascular Link' is that TBI leads to cerebrovascular dysfunction, the effects of TBI directly on this system receive scant attention. The effect of TBI on the cerebrovascular system acutely has been well characterised, with reports of biphasic opening of the BBB, firstly within a few hours post-injury and then a second opening within 3-7 days post-injury, with the degree of $\mathrm{BBB}$ alteration linked to injury severity (13). The BBB is a highly selective barrier formed by a layer of endothelial cells joined together by tight junctions including proteins such as claudins, occludin, junctional adhesion molecules, and zonula occluden proteins, which are supported by the end-feet of astrocytes (14). Transport across the $\mathrm{BBB}$ is normally highly regulated through tightly controlled mechanisms that enable the bidirectional transcellular passage of essential molecules and the efflux of potentially neurotoxic substances and waste products through expression of specific transporters (14). Depending on the severity of the initial impact, the early BBB opening may be primarily a mechanical effect or alternatively can be 
caused by alterations in the transport barriers of the BBB, with increased rates of caveolin-1 mediated transcytosis (15). A second notable disruption to the $\mathrm{BBB}$ is seen within days post-injury with an increase in endothelial caveolae and decrease in junctional adhesion and tight junction proteins. Both these events permit blood-borne factors such as thrombin, albumin, and fibrinogen to enter the brain, fuelling the inflammatory response (13). This is aided by alteration in expression of endothelial adhesion molecules, which permit the entry of blood-borne leukocytes into the brain parenchyma, which interact with brain-derived immune cells (13).

Of note, following TBI, blood vessels are particularly vulnerable to primary mechanical injury, as stretch forces predominantly affect long fibres, specifically axons and blood vessels. Mechanical stress is concentrated in the perivascular regions and the depth of the sulci $(16,17)$. This matches with the pattern of tau deposition seen post-TBI and suggests that this initial mechanical disruption may be a key initiating factor in the development of tau pathology. Indeed strain on axons leads to rupture of microtubules and release of tau, which then facilitates its phosphorylation at disease related sites (18). This phosphorylation would be enhanced by other factors which alter kinase/phosphatase activity, including oxidative stress and inflammation (2), which would be enhanced by cerebrovascular system dysfunction, particularly given the co-localisation of axonal and vascular injury (4). Surprisingly Ramos Cejudo et al. downplay the role of axonal injury in the development of $\mathrm{A} \beta$ plaque pathology immediately post-injury, focusing only on the effects of reduced blood flow and the disrupted BBB. It is known that following TBI, aggregation and deposition of $A \beta$ is markedly accelerated and can be evident within a few hours post-injury (19). However, axons are thought to be a key source of this $\mathrm{A} \beta$, as disruption of axonal transport allows accumulation of proteins in the axon, including amyloid precursor protein, with $\beta$ - and $\gamma$-secretase activity promoting $\mathrm{A} \beta$ formation (20). Notably, these are typically diffuse plaques and their number reduces over time in the acute period post-injury (21). This suggests that chronic A $\beta$ pathology is either due to an impairment in the clearance of this $A \beta$ in some individuals, or due to the development of new plaque pathology caused by ongoing secondary pathology.

Following this acute phase, it is now evident that there can be ongoing secondary injury cascades that fuel the increased risk of later developing neurodegenerative disease (16). As suggested by Ramos-Cejudo et al., it is likely that ongoing cerebrovascular dysfunction is one such factor, which feeds into other deleterious processes including chronic persistent inflammation and ongoing axonal injury (16). Although there are a paucity of studies that have investigated long-term changes to the BBB postinjury, the evidence provided by Ramos-Cejudo et al. is sparse. Utilising a focal model of TBI, the Badaut group investigated the effects of an injury in adolescence on alterations in the BBB in adulthood. Although the barrier function of the $\mathrm{BBB}$ remained intact, phenotypic changes were noted with increased expression of the tight-junction protein claudin-5 and the basement membrane proteins perlecan and fibronectin (22,23). Levels of P-glycoprotein, a key efflux pump involved in $\mathrm{A} \beta$ clearance was decreased at both 2 and 6 months post-injury (23). These changes were accompanied by an increase in $\mathrm{A} \beta$ levels compared to sham animals with increased accumulation from 2-6 months post-injury (23). Following a focal injury in adulthood, progressive $\mathrm{BBB}$ dysfunction sufficient to lead to barrier breakdown was noted, with an increase in number of microbleeds within the corpus callosum seen up to 3 months post-injury (24). This contrasts to a clinical study examining long-term survivors of TBI where $47 \%$ showed multifocal perivascular deposition of fibrinogen and immunoglobulin indicating loss of normal BBB barrier function, which was localised to the grey matter with preferential distribution towards the crests of gyri and neocortical layers (25). Notably the regions described here do not align with the pattern of tau pathology seen following TBI, where NFTs are preferentially deposited in the sulci $(3,4)$. Nonetheless, these studies suggest that ongoing alterations to the $\mathrm{BBB}$ can occur following TBI and this may differ depending on the nature of the initial insult. Alterations in the expression of structural proteins, transporters or immune receptors may occur or there may be frank discrete areas of BBB disruption. Linking these alterations to the pattern of distribution of $\mathrm{A} \beta$ and tau post-injury would provide more concrete evidence of the role of CVD post-TBI in later neurodegeneration.

In conclusion, the editorial by Ramos-Cejudo et al. is compelling and offers a thought provoking proposal of a mechanism that may account for the relationship between TBI and the subsequent development of dementia. Despite this, however, and notwithstanding the excellent evidence provided for the role of toxic $A \beta$ and tau pathology in propagating dysfunction in the cerebral vasculature, there are key pieces of experimental evidence still lacking, particularly in regards to long-term alterations in the 
$\mathrm{BBB}$ and cerebrovascular function post-injury, that must be addressed before such a hypothesis can gain a more influential place in the field.

\section{Acknowledgements}

Research supported by funding from the Neurosurgical Research Foundation and National Health and Medical Research Foundation.

\section{Footnote}

Conflicts of Interest: The authors have no conflicts of interest to declare.

\section{References}

1. Barnes DE, Byers AL, Gardner RC, et al. Association of Mild Traumatic Brain Injury With and Without Loss of Consciousness With Dementia in US Military Veterans. JAMA Neurol 2018;75:1055-61.

2. Collins-Praino LE, Corrigan F. Does neuroinflammation drive the relationship between tau hyperphosphorylation and dementia development following traumatic brain injury? Brain Behav Immun 2017;60:369-82.

3. Johnson VE, Stewart W, Smith DH. Widespread tau and amyloid-beta pathology many years after a single traumatic brain injury in humans. Brain Pathol 2012;22:142-9.

4. McKee AC, Stein TD, Kiernan PT, et al. The neuropathology of chronic traumatic encephalopathy. Brain Pathol 2015;25:350-64.

5. Braak H, Braak E. Neuropathological stageing of Alzheimer-related changes. Acta Neuropathol 1991;82:239-59.

6. Maas A. Traumatic brain injury: Changing concepts and approaches. Chin J Traumatol 2016;19:3-6.

7. Smith EE, Greenberg SM. Beta-amyloid, blood vessels, and brain function. Stroke 2009;40:2601-6.

8. Fossati S, Ghiso J, Rostagno A. Insights into caspasemediated apoptotic pathways induced by amyloid-beta in cerebral microvascular endothelial cells. Neurodegener Dis 2012;10:324-8.

9. Castillo-Carranza DL, Nilson AN, Van Skike CE, et al. Cerebral Microvascular Accumulation of Tau Oligomers in Alzheimer's Disease and Related Tauopathies. Aging Dis 2017;8:257-66.

10. Merlini M, Wanner D, Nitsch RM. Tau pathologydependent remodelling of cerebral arteries precedes
Alzheimer's disease-related microvascular cerebral amyloid angiopathy. Acta Neuropathol 2016;131:737-52.

11. Blair LJ, Frauen HD, Zhang B, et al. Tau depletion prevents progressive blood-brain barrier damage in a mouse model of tauopathy. Acta Neuropathol Commun 2015;3:8.

12. Janelidze S, Mattsson N, Stomrud E, et al. CSF biomarkers of neuroinflammation and cerebrovascular dysfunction in early Alzheimer disease. Neurology 2018;91:e867-e877.

13. Chodobski A, Zink BJ, Szmydynger-Chodobska J. Bloodbrain barrier pathophysiology in traumatic brain injury. Transl Stroke Res 2011;2:492-516.

14. Corrigan F, Mander KA, Leonard AV, et al. Neurogenic inflammation after traumatic brain injury and its potentiation of classical inflammation. J Neuroinflammation 2016;13:264.

15. Nag S, Manias JL, Stewart DJ. Expression of endothelial phosphorylated caveolin-1 is increased in brain injury. Neuropathol Appl Neurobiol 2009;35:417-26.

16. Faden AI, Wu J, Stoica BA, et al. Progressive inflammationmediated neurodegeneration after traumatic brain or spinal cord injury. Br J Pharmacol 2016;173:681-91.

17. Ghajari M, Hellyer PJ, Sharp DJ. Computational modelling of traumatic brain injury predicts the location of chronic traumatic encephalopathy pathology. Brain 2017;140:333-43.

18. Ahmadzadeh H, Smith DH, Shenoy VB. Viscoelasticity of tau proteins leads to strain rate-dependent breaking of microtubules during axonal stretch injury: predictions from a mathematical model. Biophys J 2014;106:1123-33.

19. Johnson VE, Stewart W, Smith DH. Traumatic brain injury and amyloid-beta pathology: a link to Alzheimer's disease? Nat Rev Neurosci 2010;11:361-70.

20. Uryu K, Chen XH, Martinez D, et al. Multiple proteins implicated in neurodegenerative diseases accumulate in axons after brain trauma in humans. Exp Neurol 2007;208:185-92.

21. Chen XH, Johnson VE, Uryu K, et al. A lack of amyloid beta plaques despite persistent accumulation of amyloid beta in axons of long-term survivors of traumatic brain injury. Brain Pathol 2009;19:214-23.

22. Jullienne A, Roberts JM, Pop V, et al. Juvenile traumatic brain injury induces long-term perivascular matrix changes alongside amyloid-beta accumulation. J Cereb Blood Flow Metab 2014;34:1637-45.

23. Pop V, Sorensen DW, Kamper JE, et al. Early brain injury alters the blood-brain barrier phenotype in parallel with beta-amyloid and cognitive changes in adulthood. J Cereb 
Blood Flow Metab 2013;33:205-14.

24. Glushakova OY, Johnson D, Hayes RL. Delayed increases in microvascular pathology after experimental traumatic brain injury are associated with prolonged inflammation, blood-brain barrier disruption, and progressive white

Cite this article as: Collins-Praino L, Corrigan F. Cerebrovascular contribution to dementia development after traumatic brain injury: promises and problems. Ann Transl Med 2018;6(Suppl 1):S58. doi: 10.21037/atm.2018.10.22 matter damage. J Neurotrauma 2014;31:1180-93.

25. Hay JR, Johnson VE, Young AM, et al. Blood-Brain Barrier Disruption Is an Early Event That May Persist for Many Years After Traumatic Brain Injury in Humans. J Neuropathol Exp Neurol 2015;74:1147-57. 